\title{
An Improved Outdoor Camera Calibration Method Based On EKF
}

\author{
Shaopo Zhu ${ }^{123}$, Dong $\mathrm{Hu}^{123}$ \\ ${ }^{1}$ Education Ministry's Key Lab of Broadband Wireless Communication and Sensor Network Technology \\ ${ }^{2}$ Jiangsu Province's Key Lab of Image Procession and Image Communications \\ ${ }^{3}$ Nanjing University of Posts and Telecommunications, Nanjing 210003, China \\ 1011010613@njupt.edu.cn, hud@njupt.edu.cn
}

\begin{abstract}
An improved outdoor camera calibration method based on Extended Kalman Filter (EKF) is proposed in this paper. GPS coordinate system is introduced to establish world coordinate system in the calibration procedure. According to the characteristics of GPS coordinate converted to plane coordinate and the camera calibration model, we establish the mapping relations of reference points between image plane coordinate and the world coordinate. Further, we consider the world coordinate error, set the world coordinates as the measure quantities, use the method of EKF to get the optimal estimation of intrinsic and extrinsic camera parameters, in EKF the camera's intrinsic and extrinsic parameters are set as state variables, the transformation equations of image coordinate to the world coordinate are set as the measurement equations, and we let the observed feature points' image coordinates and corresponding world coordinates as the input of the filter. Experimental results confirmed that the proposed method with high precision.
\end{abstract}

Index Terms - camera calibration, GPS coordinate, EKF, world coordinate error

\section{Introduction}

In multi-camera video surveillance system, unified world coordinate system is required to be adopted to obtain accurate target location information in outdoor environments. Therefore, GPS can be introduced in camera calibration to establish unified world coordinate system. In paper[1] Liao and Chu set GPS coordinates as world coordinates in camera calibration with Tsai's method[2]. Besides, they realized target tracking in image plane by continuously obtained GPS coordinates. Shi and $\mathrm{Hu}[3]$ further studied the problem of camera calibration with GPS coordinate system. Taking the impact of GPS receiving accuracy and target detection accuracy into consideration, they used the estimated camera parameters to project the target coordinates to the image plane again, and constructed the cost function by the sum of the differences of object's image coordinates and projected image coordinates, and then obtained optimized camera calibration parameters through GA-PSO hybrid optimization, thus improve the accuracy of the calibration. However, the final achievable calibration accuracy was limited because the overall optimization cannot be carried out in the optimization process. Therefore, in order to further improve the accuracy of camera calibration, it is necessary to find a more effective overall camera parameters estimation method to further reduce the error in the world coordinates.

Camera calibration using Kalman Filter has been paid much attention in recent years. Here, camera calibration is converted to the problem of parameter estimation. Once the relationship between observations and camera parameters is made, we could establish the state equation and the measurement equation, use the estimated value of previous time and the observe value of current time to update the state variables and thus obtain more accurate estimation of the state variables, iteratively obtain the calibration parameters. In order to solve the nonlinear of the measurement equation, Extended Kalman filter (EKF) can be used to linearizing the nonlinear equations. Many researchers have studied camera calibration using EKF. Ref. [4] and [5] treated feature points on plane calibration panel as uniformly moving points, estimate the intrinsic and extrinsic camera parameters through EKF. In [6], EKF camera calibration was used in robot vision system, the obtained camera parameters were used to estimate the exact position relationship between the specific coordinate systems.

Based on the above analysis, we propose an improved outdoor camera calibration method based on EKF. First, we transform the GPS coordinates into plane coordinates and set as absolute world coordinate, using Tsai's two-step method to get initial value of intrinsic and extrinsic camera parameters, then establish the measurement equation of EKF according to the nonlinear mapping relationship between reference point's image coordinates and the world coordinate. considering the actual measurement, there exist errors of reference points' world coordinates and image coordinates, so set them as observables, while the camera's intrinsic and extrinsic parameters are set as EKF's state variables. With the reference point's image coordinates and world coordinates as the filter input, through EKF we can finally get the optimal estimation of intrinsic and extrinsic camera parameters.

The rest of the paper is organized as follows, section 2 briefly describes the adopted camera model and the transform relationship of the coordinates, section 3 details the description of the specific algorithm of camera calibration using the EKF, section 4 gives the experimental results and the analysis. The final summary is given in section 5 .

\section{A Brief Description of the Camera Calibration Model and Method to Restore the World Coordinate}

\section{A. Camera calibration model}

As Tsai's calibration process [2] is relatively simple and can get relative good estimate of intrinsic and extrinsic camera parameters, this paper uses Tsai's camera calibration model. As is shown in Figure 1, the coordinate systems' related transformations are depicted as follows: 


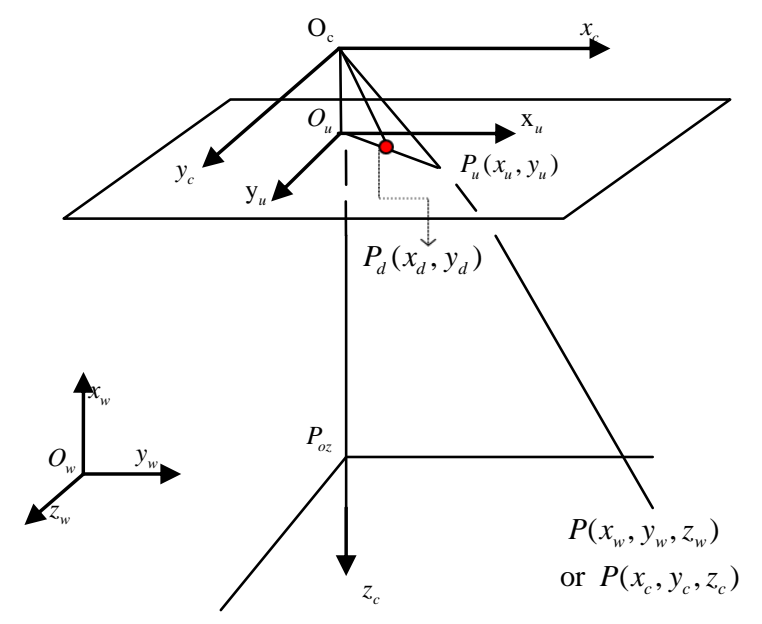

Fig. 1 Camera model

1) World coordinate transformed to camera coordinate:

$$
\left[\begin{array}{l}
x_{c} \\
y_{c} \\
z_{c}
\end{array}\right]=R\left[\begin{array}{l}
x_{w} \\
y_{w} \\
z_{w}
\end{array}\right]+T=\left[\begin{array}{lll}
r_{1} & r_{2} & r_{3} \\
r_{4} & r_{5} & r_{6} \\
r_{7} & r_{8} & r_{9}
\end{array}\right]\left[\begin{array}{l}
x_{w} \\
y_{w} \\
z_{w}
\end{array}\right]+\left[\begin{array}{l}
t_{x} \\
t_{y} \\
t_{z}
\end{array}\right]
$$

Where $\left(x_{w} y_{w} z_{w}\right)$ is the world coordinate, $\left(x_{c} y_{c} z_{c}\right)$ is the camera coordinate, $R$ and $T$ is the rotation matrix and translation matrix respectively.

2) Camera coordinate transformed to image coordinate (physical unit):

$$
x_{u}=f x_{c} / z_{c} \quad y_{u}=f y_{c} / z_{c}
$$

Where $f$ is focal length, $\left(x_{u}, y_{u}\right)$ is the ideal image point coordinate. Consider radial distortion, get the real image coordinate from the ideal image coordinate:

$$
x_{d}\left(1+k_{1} r^{2}\right)=x_{u} \quad y_{d}\left(1+k_{1} r^{2}\right)=y_{u}
$$

Where $\left(x_{d}, y_{d}\right)$ is the actual image point coordinate, $r=\sqrt{x_{d}{ }^{2}+y_{d}^{2}}, \quad k_{1}$ is the first-order radial distortion coefficient.

3) Real image coordinate transformed to the pixels image coordinate:

$$
u_{d}=s_{x} d_{x}^{-1} x_{d}+u_{0} \quad v_{d}=d_{y}^{-1} y_{d}+v_{0}
$$

Where $d_{x}^{\prime}=d_{x} N_{c x} / N_{f x}, d_{x}, d_{y}$ is the distance between two adjacent pixels in $\mathrm{x}$-and $\mathrm{y}$-direction, $s_{x}$ is the nondeterministic scale factor which is caused by camera horizontal scanning and sampling timing error, $N_{c x}$ is the CCD sensor elements in the x-direction, $N_{f x}$ is the total number of true capture pixels in $\mathrm{x}$ direction, $\left(u_{0}, v_{0}\right)$ is the main point coordinate, $\left(u_{d}, v_{d}\right)$ is the coordinate in pixels.

\section{B. Recovery the plane 2-D world coordinate from the image point coordinate}

It is pointed out in Tsai's two-step method [2], when the calibration object is 2D plane, we can get the world coordinate by backprojection of the image point coordinate, and finally by comparing the real world coordinates with the calculated world coordinates for the error analysis. The process to get the point's world coordinate from the image coordinate is described in the following.

According to the camera calibration model of this paper, when the world coordinate is plane coordinate $\left(z_{w}=0\right)$, from the conversion formula (1) of the world coordinates to the camera coordinates and the conversion formula (2) of the camera coordinates to the image coordinates, we can get the formula (5), from the formula (5) we can further derive the nonlinear transformation equation (6) of the image coordinates to the world coordinates. So when we know the intrinsic and extrinsic camera parameters, we can get the world coordinates' other two components $x_{w}$ and $y_{w}$ by the image point coordinates according to the nonlinear equation (6). The formulas are as follows:

$$
\begin{aligned}
& x_{u}=f \frac{r_{1} x_{w}+r_{2} y_{w}+t_{x}}{r_{7} x_{w}+r_{8} y_{w}+t_{z}} \quad y_{u}=f \frac{r_{4} x_{w}+r_{5} y_{w}+t_{y}}{r_{7} x_{w}+r_{8} y_{w}+t_{z}} \\
& \Rightarrow\left\{\begin{aligned}
x_{w}= & \frac{-f r_{5} t_{x}+f t_{y} r_{2}+y_{u} r_{8} t_{x}-y_{u} t_{z} r_{2}+r_{5} x_{u} t_{z}-t_{y} x_{u} r_{8}}{-x_{u} r_{7} r_{5}-r_{1} y_{u} r_{8}+r_{1} f r_{5}+x_{u} r_{8} r_{4}+r_{2} y_{u} r_{7}-r_{2} f r_{4}} \\
y_{w}= & \frac{x_{u} r_{7} t_{y}+r_{1} y_{u} t_{z}-r_{1} f t_{y}-x_{u} t_{z} r_{4}-t_{x} y_{u} r_{7}+t_{x} f r_{4}}{-x_{u} r_{7} r_{5}-r_{1} y_{u} r_{8}+r_{1} f r_{5}+x_{u} r_{8} r_{4}+r_{2} y_{u} r_{7}-r_{2} f r_{4}}
\end{aligned}\right.
\end{aligned}
$$

Note that when we know the image coordinate $\left(x_{d}, y_{d}\right)$, we still need to consider the radial distortion, according to radial distortion coefficient to calculate the ideal image point coordinate $\left(x_{u}, y_{u}\right)$, then use $\left(x_{u}, y_{u}\right)$ and the extrinsic camera parameters according to the above equations to restore the reference point's world coordinate $\left(x_{w}, y_{w}\right)$.

\section{Camera Parameters Estimation Based on EKF}

A. General process of the proposed camera calibration

General calibration flowchart is shown in Fig.2, when a moving target that carries GPS receiver enters the camera view, record the moving target's image point coordinate and GPS coordinate simultaneously. When at least 5 moving target's image point coordinates and corresponding GPS coordinates are obtained, we can get the initial value of the intrinsic and extrinsic camera parameters R, T, f, k1 by Tsai's two-step method. We should note that the measured GPS coordinate is expressed in the format of DMS(degrees-minutes-seconds) in WGS-84 coordinate system, as Beijing-54 plane coordinate is commonly used in china, we still need to transfer the coordinate from WGS-84 coordinate system into Beijing-54 plane coordinate, and set Beijing-54 plane coordinate as the world coordinate. In addition, in Tsai's two-step method the principle point coordinate is always set to be half the size of the image resolution, but the principle point coordinate is often 
deviate from the center of the image. So the principal point coordinate $(\mathrm{u} 0, \mathrm{v} 0)$ is should also be optimized. Finally, we simultaneously take $\mathrm{u} 0, \mathrm{v} 0, \mathrm{R}, \mathrm{T}, \mathrm{f}, \mathrm{k} 1$ as state variables to be optimized, and get these camera parameters' optimal estimation by EKF.

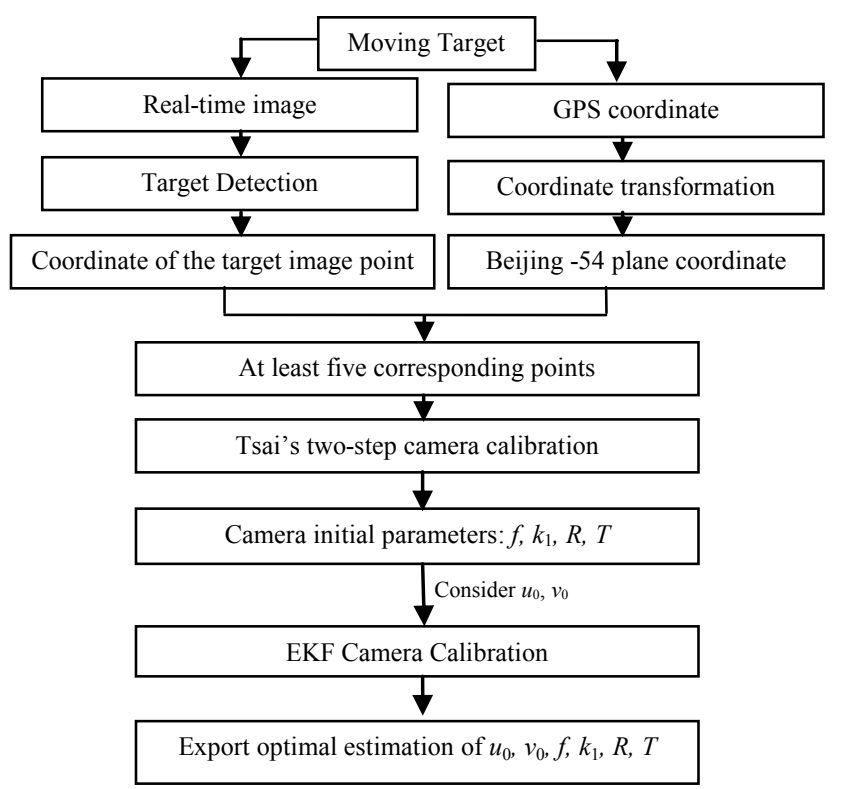

Fig. 2 General flowchart of calibration

\section{B. Camera calibration state model}

Setting the intrinsic and extrinsic camera parameters as the state parameters, the state vector is:

$$
x_{k}=\left(q_{0}, q_{1}, q_{2}, q_{3}, t_{x}, t_{y}, t_{z}, f, u_{0}, v_{o}, k_{1}\right)^{T}
$$

Wherein the rotation amount $q_{0}, q_{1}, q_{2}, q_{3}$, the shift amount $t_{x}, t_{y}, t_{z}$, the focal length is $f$, the main point coordinate is $\left(u_{0}, v_{o}\right)$, the distortion parameter is $k_{1}$. Thereby establishing the camera calibration equation of state:

$$
x_{k}=A_{k} x_{k-1}
$$

In the formula $x_{k-1}$ and $x_{k}$ are the state vector at time $k$ and $k-1$ respectively, $A_{k}$ is the state transition matrix from time $k-1$ to $k$. As long as the camera position does not change, the camera coordinate system relative to GPS's absolute world coordinate system will not change. Therefore, the camera state parameters will not change at different times, so the state transition matrix $A_{k}=I$ is a unit matrix of order 11 .

Here the rotation matrix $R$ with a unit quaternion representation.

$R=\left[\begin{array}{ccc}r_{1} & r_{2} & r_{3} \\ r_{4} & r_{5} & r_{6} \\ r_{7} & r_{8} & r_{9}\end{array}\right]=\left[\begin{array}{ccc}q_{0}^{2}+q_{1}^{2}-q_{2}^{2}-q_{3}^{2} & 2\left(q_{1} q_{2}-q_{0} q_{3}\right) & 2\left(q_{1} q_{3}+q_{0} q_{2}\right) \\ 2\left(q_{1} q_{2}+q_{0} q_{3}\right) & q_{0}^{2}-q_{1}^{2}+q_{2}^{2}-q_{3}^{2} & 2\left(q_{2} q_{3}-q_{0} q_{1}\right) \\ 2\left(q_{1} q_{3}-q_{0} q_{2}\right) & 2\left(q_{2} q_{3}+q_{0} q_{1}\right) & q_{0}^{2}-q_{1}^{2}-q_{2}^{2}+q_{3}^{2}\end{array}\right]$ (8)

Wherein $q_{0}^{2}+q_{1}^{2}+q_{2}^{2}+q_{3}^{2}=1$.

\section{Camera calibration measurement model}

In the calibration process, the feature point's image coordinate $\left(x_{u}, y_{u}\right)$ and the corresponding world coordinate $\left(x_{w}, y_{w}\right)$ can be measured, select as the observation vector, the observation model can be described as:

$$
z_{k}=\left[h_{1}(k), h_{2}(k)\right]^{T}+\left[n_{1}(k), n_{2}(k)\right]^{T}=M_{k}+N_{k}
$$

where $N_{k}$ is zero-mean white noise, $M_{k}$ can be obtained by the formula (6) of the camera projection model:

$$
\begin{aligned}
& h_{1}(k)=\frac{-f r_{5} t_{x}+f t_{y} r_{2}+y_{u} r_{8} t_{x}-y_{u} t_{z} r_{2}+r_{5} x_{u} t_{z}-t_{y} x_{u} r_{8}}{-x_{u} r_{7} r_{5}-r_{1} y_{u} r_{8}+r_{1} f r_{5}+x_{u} r_{8} r_{4}+r_{2} y_{u} r_{7}-r_{2} f r_{4}}=x_{w}(k)(10 \alpha) \\
& h_{2}(k)=\frac{x_{u} r_{7} t_{y}+r_{1} y_{u} t_{z}-r_{1} f t_{y}-x_{u} t_{z} r_{4}-t_{x} y_{u} r_{7}+t_{x} f r_{4}}{-x_{u} r_{7} r_{5}-r_{1} y_{u} r_{8}+r_{1} f r_{5}+x_{u} r_{8} r_{4}+r_{2} y_{u} r_{7}-r_{2} f r_{4}}=y_{w}(k)
\end{aligned}
$$

\section{Extended Kalman filter algorithm}

Since the measurement equation formulas $(10 \alpha)$ and $(10 \beta)$ which are obtained by camera calibration model are nonlinear equations, so this paper's calibration method is applicable to Extended Kalman filter. For a detailed description of the Extended Kalman filter we can refer to [7], this paper's Extended Kalman filter algorithm flowchart is shown in Figure 3.

1) Time update equations are:

$$
\begin{gathered}
\hat{x}_{k}^{-}=A_{k} \hat{x}_{k-1} \\
P_{k}^{-}=A_{k} P_{k-1} A_{k}^{T}
\end{gathered}
$$

Formula (11) is time update equation from time $k-1$ to time $k, \hat{x}_{k-1}$ is the state variable at time $k-1, \hat{x}_{k}^{-}$is the state variable's priori estimates at time $k$. Formula (12) obtain the priori estimate of state variable's error covariance matrix $P_{k}^{-}$at time $k, P_{k-1}$ is the state variables' error covariance matrix at time $k-1$.

2) Measurement update equations are:

$$
\begin{aligned}
& K_{k}=P_{k}^{-} H_{k}^{T}\left(H_{k} P_{k}^{-} H_{k}^{T}+R_{k}\right)^{-1} \\
& \hat{x}_{k}=\hat{x}_{k}^{-}+K_{k}\left(z_{k}-\hat{z}_{k}^{-}\right) \\
& P_{k}=\left(I-K_{k} H_{k}\right) P_{k}^{-}
\end{aligned}
$$

Where $K_{k}$ is the kalman gain, $H_{k}=\left[\partial h_{1}(k) / \partial x_{j}(k) \quad \partial h_{2}(k) / \partial x_{j}(k)\right]^{T}$ is the measurement update matrix $(10 \alpha)(10 \beta)$ relative to state variables' jacobian matrix, $R_{k}$ is Covariance matrix of the measure noise $N_{k}, z_{k}$ is the actual measured point's world coordinate, $\hat{z}_{k}^{-}$is the estimated world coordinate, $\hat{x}_{k}$ is state variables' posterior estimate at time $k, P_{k}$ is the posteriori estimates of state variables' error covariance matrix. Through observations $z_{k}$ continuously improve the estimate of state 
variables. The optimal estimation of intrinsic and extrinsic camera parameters is the state variable $x_{k}$ which is finally got by iterative.

From the principle of EKF, we know that the EKF's update on the state variables is a dynamic process. We use the measured world coordinates and image coordinates to constantly update on the estimation of state variables which are intrinsic and extrinsic camera parameters.

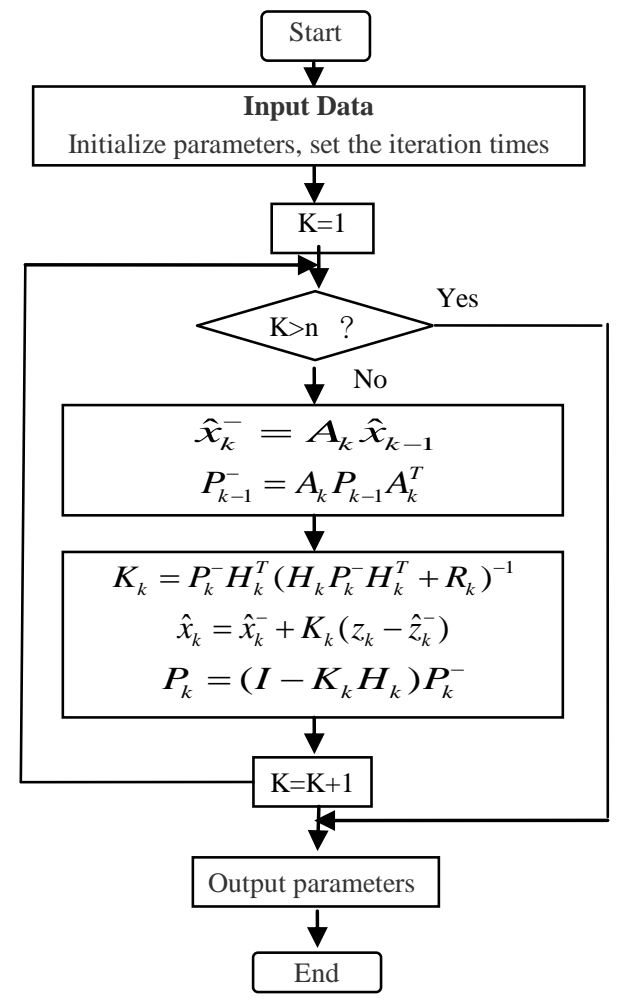

Fig. 3 EKF algorithm flowchart

\section{Simulation Results}

Camera parameters' estimation accuracy is influenced by GPS receiver's receiving accuracy and the measurement location's skyview situation. GPS receiver used in this experiment is UniStrongMG750, which is a differential GPS receiver has positioning accuracy with $1-3 \mathrm{~m}$, Camera is Sony DSC-T99 Digital Camera. We select two sites in campus (site A and B), both sites have a wide field of view and experiments carried out under good weather conditions. Figure 4 shows that in site A the measured image coordinate of a point and its corresponding transformed GPS coordinate, in the picture the transformed GPS coordinate is denoted by beijing-54 plane coordinate, GPS coordinate conversion tool is Coord.exe. The image size of the captured image sequence is $640 * 480$.

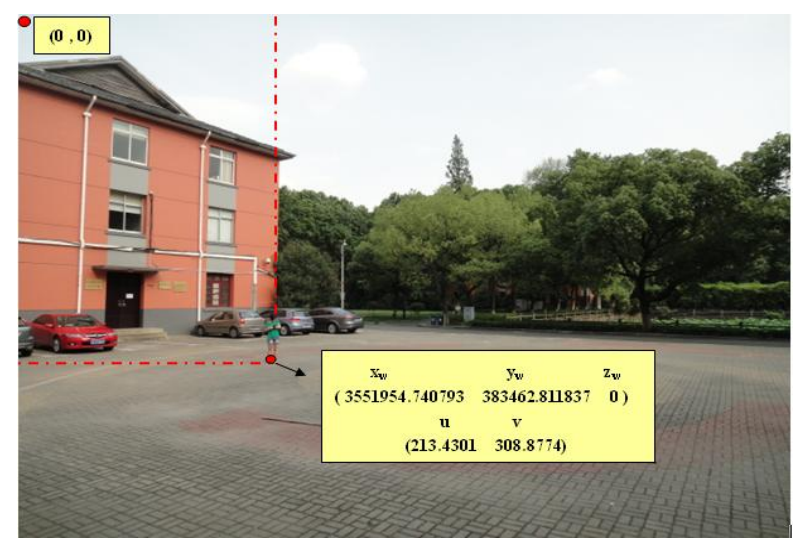

Fig. 4 The image coordinate and corresponding GPS coordinate in site A

Trials in two sites we fix the camera's position respectively, in each site we collect 15 points, these 15 points corresponding to world coordinates and image point coordinates are used for the proposed camera calibration method, to get the camera's intrinsic and extrinsic parameters. According to Tsai's camera calibration's error analysis method[2] when calibration object is planar and the linear camera calibration method's error analysis method[8]. In this paper, the error of the world coordinate is used to verify the accuracy of the obtained camera parameters. The experimental results obtained from two sites are shown in Table 1 and Table 2, where the measured world coordinate is $\left(x_{w}, y_{w}\right)$ which is obtained from the conversion of the GPS coordinate, the calculated world coordinate is $\left(x_{w}^{\prime}, y_{w}^{\prime}\right)$ which is obtained from the image point coordinate based on intrinsic and extrinsic camera parameters. $\Delta x_{w}$ is the error of $x_{w}, \Delta y_{w}$ is the error of $y_{w}$. Calculation results keep two decimal places.

TABLE I World Coordinates Error of Site A (in meters)

\begin{tabular}{|c|c|c|c|c|}
\hline & \multicolumn{4}{|c|}{ SITE A } \\
\hline $\begin{array}{c}\text { Labe } \\
1\end{array}$ & Measurement $\left(x_{w}, y_{w}\right)$ & Estimation $\left(x_{w}, y_{w}\right)$ & $\Delta x_{w}$ & $\Delta y_{w}$ \\
\hline 1 & {$[3551952.7,383461.74]$} & {$[3551962.80,383457.21]$} & 10.13 & 4.52 \\
\hline 2 & {$[3551953.1,383473.81]$} & [ 3551962.37, 383458.90] & 9.23 & 14.90 \\
\hline 3 & {$[3551955.6,383447.08]$} & {$[3551962.79,383455.34]$} & 7. 18 & 8.26 \\
\hline 4 & {$[3551964.7,383462.14]$} & [ 3551962.93, 383457.59] & 1.74 & 4.54 \\
\hline 5 & {$[$ [3551964.0, 383465.28] } & {$[3551966.46,383463.07]$} & 2.44 & 2.19 \\
\hline 6 & {$[3551963.4,383462.12]$} & {$[3551963.18,383457.22]$} & 0.25 & 4.89 \\
\hline 7 & {$[3551955.6,383447.08]$} & 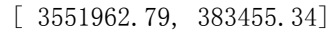 & 7. 18 & 8.26 \\
\hline 8 & {$[3551953.7,383451.26]$} & {$[3551959.35,383450.69]$} & 5.65 & 0.56 \\
\hline 9 & {$[3551952.8,383448.89]$} & {$[3551962.10,383455.30]$} & 9.29 & 6.42 \\
\hline 10 & [ 3551954.7, 383448.12] & 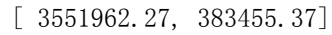 & 7. 60 & 7.25 \\
\hline 11 & {$[3551953.3,383457.02]$} & {$[3551963.15,383457.55]$} & 9.82 & 0.52 \\
\hline 12 & {$[3551959.6,383447.92]$} & {$[3551963.17,383453.68]$} & 3. 57 & 5.76 \\
\hline 13 & {$[3551962.0,383456.34]$} & {$[3551956.70,383450.92]$} & 5.26 & 5.41 \\
\hline 14 & {$[3551957.3,383459.69]$} & {$[3551960.56,383453.39]$} & 3.25 & 6.30 \\
\hline 15 & [ 3551960.1, 383453.69] & [ 3551964.90, 383459.68] & 4. 75 & 5.99 \\
\hline
\end{tabular}


TABLE II World Coordinates Error of Site B (in meters)

\begin{tabular}{|c|c|c|c|c|}
\hline & \multicolumn{4}{|c|}{ SITE B } \\
\hline $\begin{array}{c}\text { Labe } \\
1\end{array}$ & Measurement $\left(x_{w}, y_{w}\right)$ & Estimation $\left(x_{w}^{\prime}, y_{w}^{\prime}\right)$ & $\Delta x_{w}$ & $\Delta y_{w}$ \\
\hline 1 & [ 3551958.1, 383457.97] & {$[3551954.16,383464.82]$} & 3.96 & 6.85 \\
\hline 2 & [ 3551953.9, 383454.15] & {$[3551953.37,383463.70]$} & 0.54 & 9. 55 \\
\hline 3 & {$[3551944.5, \quad 383466.0]$} & {$[3551954.62,383464.35]$} & 10.08 & 1.64 \\
\hline 4 & {$[3551944.1,383475.12]$} & [ 3551954.28, 383464.52] & 10.21 & 10.59 \\
\hline 5 & {$[3551944.8,383460.65]$} & 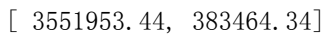 & 8.65 & 3. 69 \\
\hline 6 & {$[3551952.0,383459.58]$} & [ 3551954.29, 383459.96] & 2.28 & 0.38 \\
\hline 7 & {$[3551953.8,383461.23]$} & [ 3551954.35, 383464.62] & 0.51 & 3. 39 \\
\hline 8 & {$[3551953.5,383460.91]$} & {$[3551954.77,383464.47]$} & 1.30 & 3.56 \\
\hline 9 & {$[3551955.6,383465.65]$} & [ 3551953.84, 383464.49] & 1.79 & 1. 16 \\
\hline 10 & {$[3551952.6,383470.66]$} & [ 3551953.79, 383464.68] & 1.17 & 5.97 \\
\hline 11 & {$[3551951.1,383476.78]$} & {$[3551953.77,383464.75]$} & 2.69 & 12.02 \\
\hline 12 & {$[3551952.0,383463.09]$} & {$[3551953.83,383464.85]$} & 1.86 & 1.75 \\
\hline 13 & {$[3551954.7,383462.81]$} & {$[3551953.83,383464.86]$} & 0.91 & 2.05 \\
\hline 14 & {$[3551952.7, \quad 383463.1]$} & {$[3551953.85,383464.87]$} & 1.14 & 1.76 \\
\hline 15 & {$[3551956.2, \quad 383460.63]$} & 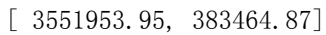 & 2.29 & 4. 24 \\
\hline
\end{tabular}

In this paper we calculate the standard deviation of the world coordinates error for all points in site A and site B. And we compare the results with Tsai's two-step method which is used in [2] and the results get by GA-PSO hybrid optimization algorithm which is used in Shi' camera calibration method[3]. Table 3 shows the result of the comparison, the proposed refers to the calibration error results which are calculated by this paper's camera calibration parameters. Calculation results keep two decimal places, units are meters.

TABLE III Standard Deviation of Site A and Site B (in standard deviation tests)

\begin{tabular}{|c|c|c|c|}
\hline \multirow{2}{*}{} & $\sigma_{x w}$ & $\sigma_{y w}$ \\
\hline \multirow{4}{*}{ Site A } & Tsai' & 6.17 & 10.77 \\
\cline { 2 - 4 } & Shi' & 5.77 & 5.92 \\
\cline { 2 - 4 } & proposed & 3.13 & 3.48 \\
\hline \multirow{3}{*}{ Site B } & Tsai' & 10.66 & 8.90 \\
\cline { 2 - 4 } & Shi' & 4.97 & 6.08 \\
\cline { 2 - 4 } & proposed & 3.42 & 3.65 \\
\hline
\end{tabular}

TABLE IV The Impact of GPS Receiver's Receiving Accuracy on The Camera Calibration Accuracy (in standard deviation tests)

\begin{tabular}{|c|c|c|c|}
\hline \multirow{2}{*}{} & $\sigma_{x w}$ & $\sigma_{y w}$ \\
\hline \multirow{2}{*}{ UniStrongMG750 } & siteA & 3.13 & 3.48 \\
\cline { 2 - 4 } & siteB & 3.42 & 3.65 \\
\hline \multirow{2}{*}{ UniStrongMG838 } & siteA' $^{\prime}$ & 0.45 & 1.84 \\
\cline { 2 - 4 } & siteB' & 0.63 & 2.17 \\
\hline
\end{tabular}

Table 1 and Table 2 list the error of world coordinates of site A and site B respectively, this paper's calibration method can be seen with relatively good accuracy, the camera calibration parameters obtained can accurately restore the world coordinates. It can be seen in Table 3 the comparison of the proposed method with other two methods, the world coordinates recovered by proposed method have smaller errors, and camera calibration accuracy has a greater degree of improvement compared with other two methods. To test the impact of GPS receiver's receiving accuracy on the camera calibration accuracy, this paper use another differential GPS receiver UniStrongMG838(positioning accuracy is about 1 meter) conduct camera calibration by this paper's calibration method, and compare the standard deviation of the world coordinates error with UniStrongMG750(positioning accuracy is 1-3 meter) which is used above, Table 4 shows the comparison results of two GPS receiver, from the comparison results we can see UniStrongMG838 have smaller errors, proved that improve the GPS receiver's receiving accuracy can improve the camera calibration's accuracy.

\section{Summary}

In this paper we proposed a new outdoor camera calibration algorithm based on EKF. To meet the requirement for outdoor application, we also start from Tsai's two-step algorithm combining GPS to establish world coordinate system. Detailed procedure to establish measurement equation and state equation are described, which are based on the projection relation from image plane and world coordinate system. Both intrinsic and extrinsic camera parameters are estimated iteratively by EKF optimally. Experimental results show that the camera parameters obtained by the proposed algorithm possess relative high precision, and prove the effectiveness of the proposed method.

\section{Acknowledgment}

This work was supported by the Natural Science Grand Research Project of Jiangsu province (Grant No. 10KJA510036), and also by the Project of the Priority Academic Program Development of Jiangsu Higher Education Institutions: Information and Communication Engineering.

\section{References}

[1] H.C.Liao, P.T.Chu, "A Novel Visual Tracking Approach Incorporating Global Positioning System in a Ubiquitous Camera Environment", Information Technology Journal 8 (4), pp. 465-475, 2009.

[2] R.Y. Tsai, "A versatile camera calibration technique for high-accuracy 3D machine vision metrology using off-the-shelf TV cameras and lenses", IEEE Journal of Robotics and Automation, vol. 3, no. 4, 1987, pp. 323-344.

[3] Y.J.Shi, D.Hu, "Outdoor Camera Calibration Method Using GPS Coordinate Based On Two-stage Optimization", Image Analysis and Signal Processing (IASP), 2012 International Conference on. IEEE, 2012, pp. 1-5.

[4] G.J. Zhang. "Vision measurement". Beijing: Science Press, 2008.

[5] Y.X.Zhu, X.S.Duan, "A Method of Traditional Camera Calibration Considering Distortion based on Extended Kalman Filter", International Symposium on Computational Intelligence and Design, 2011,pp.178181.

[6] G.Chen, Z.Xia, M.Xie, "Camera Calibration based on Extended Kalman Filter using Robot's Arm Motion", IEEE/ASME International Conference on Advanced Intelligent Mechatronics, 2009, pp.1839-1844.

[7] G.Welch, G.Bishop, "An Introduction to the Kalman Filter", 1997.

[8] W.C.Zhang, J.P.Mei, Y.B.Ding, "A Simple Camera Calibrating Method Based on Local linearization Technology", International Conference on Environmental Science and Information Application Technology 10 ( 2011 ), pp. $269-274$. 